\title{
Modeling of Low Coherence Interferometry Using Broadband Multi-Gaussian Light Sources
}

\author{
Paul JANSZ*, Steven RICHARDSON, Graham WILD, and Steven HINCKLEY \\ School of Engineering, Edith Cowan University, 270 Joondalup Drive, Joondalup WA 6027, Australia \\ *Corresponding author: Paul JANSZＥ-mail: p.jansz@ecu.edu.au
}

\begin{abstract}
Using a low coherence interferometry (LCI) model, a comparison of broadband single-Gaussian and multi-Gaussian light sources has been undertaken. For single-Gaussian sources, the axial resolution improves with the source bandwidth, confirming the coherence length relation that the resolution for single Gaussian sources improves with increasing spectral bandwidth. However, narrow bandwidth light sources result in interferograms with overlapping strata peaks and the loss of individual strata information. For multiple-Gaussian sources with the same bandwidth, spectral side lobes increase, reducing A-scan reliability to show accurate layer information without eliminating the side lobes. The simulations show the conditions needed for the resolution of strata information for broadband light sources using both single and multiple Gaussian models. The potential to use the model to study optical coherence tomography (OCT) light sources including super luminescent diodes (SLDs), as reviewed in this paper, as well as optical delay lines and sample structures could better characterize these LCI and OCT elements. Forecasting misinformation in the interferogram may allow preliminary corrections. With improvement to the LCI-OCT model, more applications are envisaged.
\end{abstract}

Keywords: Medical imaging, optical coherence tomography, Gaussian light sources, modelling

\section{Introduction}

Optical coherence tomography (OCT) is a medical imaging technique that is fundamentally an application of low coherence interferometry [1-3].

In previous research [4], an OCT model was used to compare different optical delay lines (ODLs) in order to verify the functionality of a proposed stationary ODL [5]. To further investigate OCT operation characteristics, the OCT model has been used to characterize one to four combined Gaussian broadband light sources which mimic the spectral characteristic of super luminescent diodes (SLDs), a typical OCT light source.

\section{Optical coherence tomography}

In this section, we provide a brief overview of the processes and method of OCT, as well as providing an introductory review of the super luminescent diode OCT light source that is relevant to, and predictive of, appropriate applications of the OCT model simulator in this paper and in future research. This research considers combinations of SLD-like sources and their resulting interferograms.

\subsection{OCT method}

In LCI and OCT, a low coherent/broadband light source is used to generate a reflection intensity map of a material's (LCI) or tissue's (OCT) cross section.

Received: 8 June 2012 / Revised version: 16 June 2012

(C) The Author(s) 2012. This article is published with open access at Springerlink.com 
In vivo (OCT), the sources penetration depth can vary from $1 \mathrm{~mm}$ to $3 \mathrm{~mm}$ or more. This depends on the tissue type as well as an increase in the longer wavelength and optical power [6]. The axial resolution can be $1 \mu \mathrm{m}$ to $10 \mu \mathrm{m}$ depending on the light source spectral shape [7-8]; decreasing in the value with the bandwidth and increasing in the value with the central wavelength [3].

Four key elements impact on the OCT detector signal: the light source, the ODL of the reference arm, the type of the interferometer used, and the sample characteristics.

The light source is low-coherent in that it has a broad frequency bandwidth. All the light frequencies interfere with each other, resulting in a self modulated light source where the width of the individual peak in the time domain is proportional to the image axial resolution, measured by the coherence length $\left(L_{\mathrm{C}}\right)$ of the source [see (1)] divided by the average tissue refractive index. The $L_{\mathrm{C}}$ can be determined from the spectral characteristics of the source using

$$
L_{\mathrm{C}}=\frac{\ln 4 \cdot \lambda_{0}^{2}}{\pi \cdot \Delta \lambda}
$$

where $\lambda_{0}$ is the source central wavelength, and $\Delta \lambda$ is the spectral full width at the half maximum (FWHM) of the power spectrum, assuming it has a Gaussian spectral profile. Typical OCT sources have $\lambda_{0}$ at $840 \mathrm{~nm}$ and $1320 \mathrm{~nm}$, with $\Delta \lambda$ equal to $60 \mathrm{~nm}$. From (1), these values give coherence lengths of $5.2 \mu \mathrm{m}$ and $12.8 \mu \mathrm{m}$, respectively.

Since the inverse Fourier transform of a perfectly Gaussian spectrum in the frequency domain is itself a Gaussian in the time domain, a Gaussian spectrum will have one peak and no repeated peaks, ideal for stratified samples. The less Gaussian that the source spectrum is in the frequency domain, the more frequently that peaks appear in the time domain. An interferometer can then be used to manipulate the introduced light to acquire an interference pattern of the multi-layered sample's reflected beam and the reflected reference ODL beam at the detector (Fig. 1).

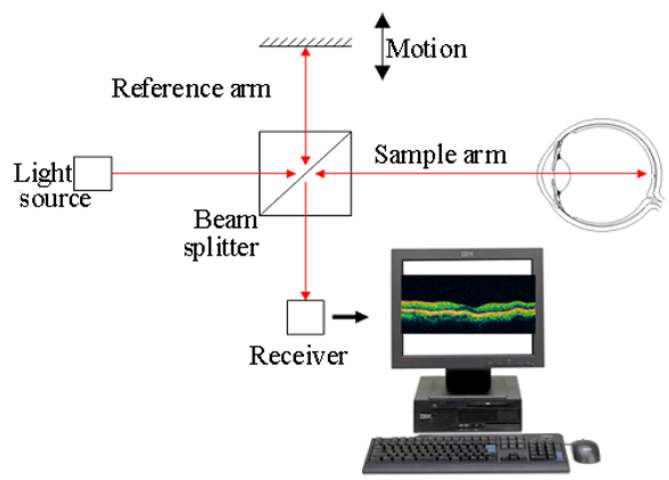

Fig. 1 Operating principle of a Michelson interferometer type OCT system.

\subsection{OCT light source characteristics}

The suitability of the optical source is governed by its bandwidth, optical power, spectral shape, ripple, and cost. Figure 2 shows the relationship between the $L_{\mathrm{C}}$ (axial resolution), FWHM bandwidth $(\Delta \lambda)$, and central wavelength $\left(\lambda_{0}\right)$.

The longer the source wavelength $\left(\lambda_{0}\right)$ is, the wider the bandwidth $(\Delta \lambda)$ is needs to be to achieve equivalent axial resolution. The wider the bandwidth is, the better the axial resolution $\left(R_{\text {axial }}\right)$ is, which can be determined from the $L_{\mathrm{C}}$ at the average tissue refractive index $\left(n_{\text {ave }}\right)$ :

$$
R_{\text {axial }}=\frac{L_{\mathrm{C}}}{n_{\text {ave }}}=\frac{\lambda_{0}^{2} \cdot \ln (4)}{\pi \cdot \Delta \lambda \cdot n_{\text {ave }}} .
$$

The larger the optical powerm is, the greater the sensitivity (limited by the biological safety irradiation limit) is. This is especially true for full field OCT where the source power is spread over a large illumination area.

The shape of the coherence function and the level of the side lobes are determined by the spectral shape. A perfect Gaussian spectrum has no side lobes in the autocorrelation function and is the preferred source spectrum. Due to their affordability and Gaussian spectral shape, OCT sources are typically SLDs. To increase the bandwidth, thus improving the axial resolution, it is common to 
combine two or more SLDs. This often leads to non-Gaussian spectra with multiple spectral peaks degrading the A-scan with the addition of the spurious smaller "side lobe" peaks [8].

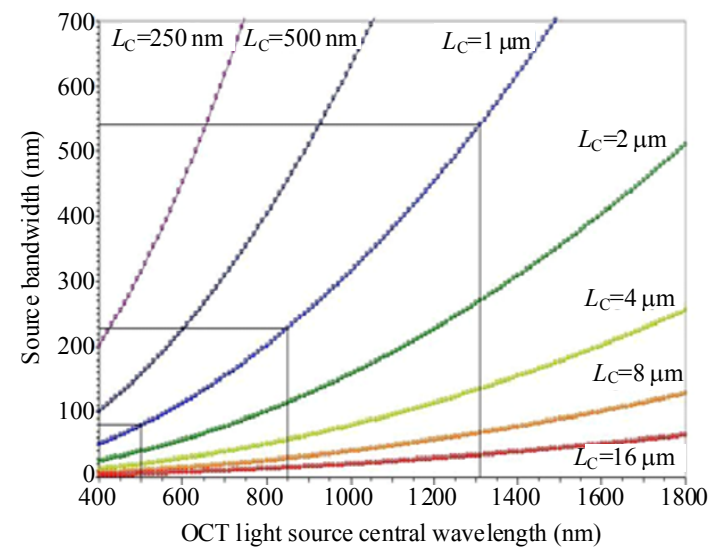

Fig. 2 Free space OCT iso-resolution lines (iso-LC lines); bandwidths for typical OCT sources (generation based on Fig. 8.2 of [2]).

\subsection{Super luminescent diode}

Since this paper considers the simulation of OCT interferograms using simulated single and tandem SLD (Gaussian) light sources, an introductory review of the SLD as an OCT source is appropriate. This review outlines the various techniques used to broaden the SLD spectra, so that the OCT axial resolution can be improved.

SLDs work like a forward biased PN junction for which a large forward bias produces a large density of electron-hole recombination and resulting light emission. The structure and operation of SLDs are similar to an edge emitting laser diode without the feedback or cavity so that lasing is prevented. Due to this high injection current, the spontaneous emission is amplified, resulting in super-luminescence. However, the higher the gain is, the narrower the spectral width is, until radiation amplification exceeds the loss, resulting in lasing, with loss of the spectral bandwidth [9]. For this reason, the current needs to be kept at a maximum value at which super-luminescence, not lasing, is generated [10]. Therefore, the SLD spectral width is between that of a light emitting diode (LED) and a laser diode.

The SLD wavelength and bandwidth are determined by the type of semiconductor materials and their layering in the waveguide structure of the diode [9]. The narrow emission angle of SLDs allows optimum coupling into the single-mode fiber for optical fiber based OCT [11]. SLDs can be manufactured to illuminate within and beyond the "therapeutic window", which is between $800 \mathrm{~nm}$ and $1350 \mathrm{~nm}[9]$.

In the earliest OCT systems, LEDs were also popular. These gave broader bandwidths than SLDs but with orders of magnitude less power in the single spatial mode, though the edge-emitting LED had higher intensity [12]. An edge emitting LED, used for high-resolution reflectometry in the biological tissue at the wavelength of $1300 \mathrm{~nm}$, bandwidth of $60 \mathrm{~nm}$, and $L_{\mathrm{C}}$ of $12.4 \mu \mathrm{m}$, produced an optical power of only $1 \mu \mathrm{W}$ [13]. Combined LED sources, at $1240 \mathrm{~nm}$ and $1300 \mathrm{~nm}$, improved the $L_{\mathrm{C}}$ from $10.8 \mu \mathrm{m}$ to $7.2 \mu \mathrm{m}$ [14]. Though the power increased 100 fold from [13], it was still only 100 $\mu \mathrm{W}[9]$.

Interest in SLDs was initiated due to their application to fiber-optic gyroscopes in the early 1980s [15] and other fiber-optic-based sensors used in navigation [16]. This development led to their use in other sensing systems including Faraday-effect electric current sensors and distributed Bragg-grating sensor systems [7]. This was soon followed in the 1990s with their application to the testing of telecommunications wavelength division multiplexing systems [17] and various low coherence interferometry systems including OCT due to their small size, ease of use, low cost, and ten-fold improvement $(10 \mathrm{~mW})$ in the output power at that time [7].

\subsection{SLD spectral broadening}

SLDs would be the OCT light source of choice 
for application to making OCT affordable to medical general practice if their bandwidth could increase without significant loss to their Gaussian spectral shape. The spectrum of an SLD is determined by its optical gain spectrum.

The first SLDs were based on bulk semiconductor hetero-structures with thick active layers. Their optical spectrum width varied from $15 \mathrm{~nm}-20 \mathrm{~nm}$ for $800-\mathrm{nm}$ AlGaAs emitters to $30 \mathrm{~nm}-40 \mathrm{~nm}$ for $1300-\mathrm{nm}-1550-\mathrm{nm}$ InGaAsP SLDs. Broadening the spectrum was attempted for these bulk active layer SLDs by including a "stacked-layer-SLD" with two active layers of different materials [7].

The most significant progress in spectral broadening was made with the demonstration of quantum-well SLDs.

\subsubsection{Quantum Well SLDs}

In a quantum well (QW) SLD, the active region of the device is so narrow that quantum confinement occurs. The wavelength of the light emitted by a quantum well source is determined by the width of the active region rather than just the bandgap of the material from which it is constructed. This means that the wavelength emitted can be tailored to a particular value. The physical form can be achieved using epitaxial growth techniques (molecular beam epitaxy) to grow a sequence of direct band-gap semiconductor layers so that the narrower bandgap material is sandwiched between the larger bandgap materials. A quantum "well" is then formed in both the valence and conduction bands. Harmonic-like, sub energy bands exist in these wells [18]. These extra energy levels can broaden the emission spectrum. The efficiency of a quantum well SLD is also greater than a conventional SLD source due to the stepwise form of its density of states function caused by introducing the well.

QW SLD spectral broadening can be achieved in two ways. Firstly, the optical gain of these SLDs is broadened by their higher density of states, compared to bulk SLDs. Secondly, transition from different sub-bands in quantum-well active layers, can be used to broaden the SLD spectrum [7]. This broadening is dependent on the magnitude of the drive current. However, as broadening increases, the spectrum can become bi-lobed [16], resulting in expected spurious side lobes appearing in the interferogram.

\subsubsection{Multiple QW SLDs}

An extension of the QW SLD, that increases spectral bandwidth, is the multiple QW (MQW) SLD in which quantum wells of various depths are created thereby broadening the emission spectrum. For example, a double QW separate confinement double hetero-structure SLD, has been demonstrated [(InGa)As/(GaAl)As/GaAs]. The combined spectra has a central wavelength of $1029.6 \mathrm{~nm}$, a bandwidth of $108.5 \mathrm{~nm}\left(L_{\mathrm{C}}=4.3 \mu \mathrm{m}\right)$, and a single mode power of $18.3 \mathrm{~mW}$ [19].

Compositionally modulated MQW SLDs have also broadened the SLD spectrum. Here, the depth of the well systematically decreases by varying the percentage compositions of the paired III-V direct bandgap semiconductor constituting each well. A bandwidth of $91 \mathrm{~nm}$ at $1300 \mathrm{~nm}$ and output power of $2 \mathrm{~mW}$ has been demonstrated [20]

\subsubsection{Quantum dots-in-well SLDs}

SLDs have been demonstrated, which are based on multiple layer "dots-in-the-wells" (DWELL) separate confinement double hetero-structures. These SLDs have a median wavelength of $1160 \mathrm{~nm}$ and bandwidth of over $100 \mathrm{~nm}\left(L_{\mathrm{C}}<5.9 \mu \mathrm{m}\right)$. However, the spectrum has multiple peaks resulting in side lobes in the coherence spectrum [19].

An addition to the MQW structure that broadens the emission spectrum of the SLD is the use of chirped quantum dot (QD) multilayers. The broadening is controlled by a change in the matrix 
surrounding the QD; that is, when the QDs are covered by an InGaAs strain-reducing layer. Depending on the thickness and composition of the strain-reducing layer, an emission red shift occurs [21]. The combination of the ground state and excited state emissions results in a bandwidth of $121 \mathrm{~nm}$, centered at $1290 \mathrm{~nm}\left(L_{\mathrm{C}}=6.1 \mu \mathrm{m}\right)$. An increasing in the injection current will increase ground state emissions and blue-shift the spectrum [21]. Similar SLDs at a wavelength of $1144 \mathrm{~nm}$ with a bandwidth of $110 \mathrm{~nm}\left(L_{\mathrm{C}}=5.2 \mu \mathrm{m}\right)$ and a power of $58 \mathrm{~mW}$, and at a wavelength of $1050 \mathrm{~nm}$ with a bandwidth of $70 \mathrm{~nm}\left(L_{\mathrm{C}}=6.9 \mu \mathrm{m}\right)$ and a power of 2 $\mathrm{mW}$ [23], have been demonstrated.

A broadening of the emission for a 1.3- $\mu \mathrm{m}$ QD well SLD can also be achieved by incorporating the InAs QDs in InGaAs QWs of the chirped indium composition. This dots in a compositionally modulated well (DCMWELL) SLD generates a broader and flatter emission compared to the DWELL SLD, which have wells of the identical indium composition and present multiple Gaussian peak spectra. This flatter emission is due to the overlapping of excited and ground state transitions from different DWELLs that have different indium well compositions [24]. An improved $3 \times 2$ chirped DCMWELL SLD with a bandwidth of $95 \mathrm{~nm}$ at the same wavelength of $1270 \mathrm{~nm}$ and power of $8 \mathrm{~mW}$, and the possibility of the output power being increased to $42 \mathrm{~mW}$ has also been demonstrated [25].

\subsubsection{Quantum dash SLDs}

A variation in the $\mathrm{QD}$ is the quantum dash (QDash), which is a finite-length wire-like structure with the height and width similar to a QD and the length much longer than that of the QD. Bandwidths up to $300 \mathrm{~nm}$ [26] and near-Gaussian emission have been reported, though only above the "therapeutic window" at around $1600 \mathrm{~nm}$. Broadening of QDash laser emission can be achieved monolithically by spatial bandgap engineering techniques employing regrowth, selective area epitaxy, or quantum heterostructure intermixing [27]. It may be possible, by using suitable combinations of larger direct bandgap semiconductor materials, to fabricate a QDash SLD that blue shifts the amplified stimulated emission spectrum into the therapeutic window while still keeping the bandwidth and other advantages of the QDash SLDs.

\subsubsection{Tandem SLDs}

Though the previous SLD spectra can be simulated using the present OCT model, this introductory paper considers the following method of increasing spectral bandwidth. Here, SLDs are optically combined so that their spectra overlap. This can be achieved by physically connecting multiple SLDs with their outputs coupled optically.

An early example of tandem SLDs had two SLDs at $830 \mathrm{~nm}$ (26-nm bandwidth) and $855 \mathrm{~nm}$ (25-nm bandwidth), optically combined with a beam splitting cube. This resulted in a reduction in the coherence length from $12 \mu \mathrm{m}$ to $8 \mu \mathrm{m}$ [28]. Wang [29] showed another multi-SLD example, which was a combination of four SLDs with central wavelength/bandwidth combinations of $1265 \mathrm{~nm} / 30 \mathrm{~nm}$, $1320 \mathrm{~nm} / 70 \mathrm{~nm}, 1355 \mathrm{~nm} / 53 \mathrm{~nm}$, and $1380 \mathrm{~nm} / 47 \mathrm{~nm}$. The combined bandwidth produced was $145 \mathrm{~nm}$ centered at $1330 \mathrm{~nm}$, having an output power of $10 \mathrm{~mW}$ and a coherence length of $5.4 \mathrm{~m}$. Other novel tandem-SLD sources are the BroadLighter D-890 (2 SLDs), Q-940, and Q-1350 (4 SLDs) with bandwidth of $150 \mathrm{~nm}, 300 \mathrm{~nm}$, and $280 \mathrm{~nm}$, respectively [19], resulting in coherence lengths of $2.3 \mu \mathrm{m}, 1.3 \mu \mathrm{m}$, and $2.9 \mu \mathrm{m}$, respectively. An example of a quantum-well double SLD was the DenseLight DL-BD9-CS31159A, a 25-mW SLD with a bandwidth of $170 \mathrm{~nm}$ at $1310 \mathrm{~nm}$, and with the $L_{\mathrm{C}}$ of $4.4 \mu \mathrm{m}$ [30]. All of these SLDs can be generated by this model to investigate their A-scans.

\subsection{Characterizing SLD OCT interferograms}

This research considers the latter spectral broadening techniques. It compares the effect of 
combining multiple broadband Gaussian sources on their total spectrum, as well as characterizing the effect on the resultant simulated interferogram, and the corresponding limitations for practical implementations. Importantly, this is for the same five-layer 1D virtual sample structure, enabling the direct comparison between SLD combinations and individual broad and narrow spectra.

Multiple SLD bandwidth broadening is not only the broadening technique. While other techniques produce wider bandwidths, multiple spectral peaks typically occur. This initial investigation demonstrates the relation between the bandwidth $(\Delta \lambda)$ and axial resolution $\left(R_{\text {axial }}\right)$ for a given central wavelength $\left(\lambda_{0}\right)$. It further compares the effects of bandwidth on the spectrum and autocorrelation function for combined Gaussian sources, corroborating current knowledge, as well as demonstrating the effect of the bandwidth and the number of Gaussian sources used.

\section{Theory}

The OCT circuit modeled is a Michelson interferometer, e.g. Fig. 1. The light source is a low coherent virtual source that can model either an SLD Gaussian spectral shape, a predefined combined spectra, or the ability to simulate A-scans using spectral curves generated by real light sources.

The sample is a stratified 1D virtual model with the definable layer depth and refractive indices. From these defined refractive indices, the layer reflectivities are calculated by the model and used to generate the complex sample reflections.

The optical delay line is a moving mirror on a linear scanner, where its travel length is defined over the total depth of the virtual sample. Other ODL can be tested, including the stationary stepped mirror ODL as well as other quasi-stationary alternatives.

\subsection{Light source}

The light source is modelled as a spectrum of continuous wavelengths, with a Gaussian spectral shape defined by the peak amplitude $\left(A_{0}\right)$, the peak wavelength $\left(\lambda_{0}\right)$, and the spectral bandwidth FWHM $(\Delta \lambda)$, as

$$
A(\lambda)=A_{0} \operatorname{Exp}\left(\frac{-\ln (16)\left(\lambda-\lambda_{0}\right)^{2}}{(\Delta \lambda)^{2}}\right) .
$$

\subsection{Light in the interferometer}

In the interferometer, light is split by a beam splitter (Fig. 1); $50 \%$ is reflected $(R)$ to the ODL, and $50 \%$ is transmitted $(T)$ to the sample. The light amplitude after the split is

$$
A_{R}(\lambda)=A_{T}(\lambda)=\frac{A(\lambda)}{\sqrt{2}} .
$$

The amplitudes after reflection from the sample interfaces are given by

$$
A_{T}(\lambda)_{i}=\frac{A(\lambda)}{\sqrt{2}} \sqrt{r_{i}} \prod_{j=1}^{i-1}\left(1-r_{j}\right)
$$

where $i=1, \ldots, n$ is the index over the semi-reflector interfaces of the sample, and $r_{i}$ denotes the reflectivity of the $i$ th interface. The total distance from the laser source to the $i$ th reflecting sample interface and then back to the detector is denoted by $d_{i}$. The model does not consider multiple reflection, due to the small reflection coefficients. However, this consideration can be included in future versions.

The ODL is a moving reference reflector, assumed to be moving slow enough to neglect the Doppler effect. The distance from the laser source to the ODL reflector and back to the beam-splitter and then to the detector is denoted by $d_{n+1}$. If the ODL reflector moves an amount $\Delta d$, then $d_{n+1}$ increases by an amount of $2 \Delta d$.

\subsection{Light at the detector - wave interference}

The square of the amplitude of the auto-correlated wave is

$$
[\text { Amplitude }(\lambda)]^{2}=\frac{A(\lambda)^{2}}{4}\left[\begin{array}{l}
\left(\sum_{i=1}^{n+1} R F_{i} \cos \theta_{i}\right)^{2} \\
+\left(\sum_{i=1}^{n+1} R F_{i} \sin \theta_{i}\right)^{2}
\end{array}\right]
$$

where 


$$
R F_{i}=\left\{\begin{array}{cc}
r_{i} \prod_{j=1}^{i-1}\left(1-r_{j}\right), & i=1, \ldots, n \\
1, & i=n+1
\end{array}\right.
$$

and

$$
\theta_{i}=\left\{\begin{array}{cc}
\frac{2 \pi d_{i}}{\lambda}+\pi, & n_{i}<n_{i+1} \\
\frac{2 \pi d_{i}}{\lambda}, & n_{i}>n_{i+1} \text { or } i=n+1
\end{array} .\right.
$$

Therefore, the total intensity is

$$
\text { Total intensity }\left(d_{n+1}\right)=\int_{-\infty}^{\infty}[\operatorname{Amplitude}(\lambda)]^{2} d \lambda \text {. }
$$

The integral in (9) is approximated numerically.

\section{Method}

In this section, the tissue model is defined, and the application of the OCT model simulator is outlined.

\subsection{Tissue model parameters}

The layered virtual sample employed in the simulation consisted of five layers with equal thickness, being $100 \mu \mathrm{m}$ and $95 \mu \mathrm{m}$ in Sections 5.1 and 5.2, respectively. This thickness is analogous to that of tissue lamina in vivo [31]. The refractive index assigned to the medium above the uppermost stratum was 1.35 . The assigned refractive indices of the five sample layers, the uppermost layer first, were $1.45,1.49,1.45,1.49$, and 1.45 , respectively. Even though these values are within biological tissue ranges, 1.37-1.5 [31], the model may be used to explore any virtual multi-strata reflecting material.

\subsection{OCT model simulator 3.0}

The flexibility of this version (3.0) of the OCT simulation model to allow, at this stage, choice of two sample layer characteristics - thickness and refractive index - enables approximate definition of real axial tissue characteristics. This then generates an autocorrelation function with twice the layer separation. For a user defined light source spectrum and ODL, the effects of OCT sources, ODLs [4], and sample types on OCT operation, can be studied to a degree remembering that a real system presents imperfections at every point in the interferometric circuit.

\section{Results and discussion}

In this section, we present results for OCT interferograms using (1) a single SLD Gaussian source with the varying bandwidth and (2) multiple SLD Gaussian sources with the constant bandwidth.

\subsection{Single source with the varying bandwidth}

Figure 3 shows the effect of Gaussian source bandwidth on the layer resolution. As the bandwidth increases, resolution increases. Note that side lobes are absent from the interferograms in Fig3. 3(b), 3(c), and 3(d). It is not possible to resolve individual layers in Fig. 3(a) because the $L_{\mathrm{C}}$ is $106 \mu$, and the layer thickness is only $100 \mu \mathrm{m}$, which is $0.94 L_{\mathrm{C}}$. In contrast, the broadest source has the best resolution because the $L_{\mathrm{C}}$ is ten times less for the same $100-\mu \mathrm{m}$ interface separation, which is $9.4 L_{\mathrm{C}}$. Even with Figs. 3(b) and 3(c), the layers can be resolved, their separations being $1.9 L_{C}$ and $4.7 L_{C}$, respectively. The hidden peak information in Fig.3(a) can lead to loss of layer information in the A-scans.

\subsection{Multiple source with constant bandwidth}

Figures 4, 5, and 6 show the interferograms for two, three, and four combined Gaussian sources, respectively. Each spectral band width is $100 \mathrm{~nm}$.

If the resolution is a function of the coherence length, Figs. 4, 5, and 6 should be as equally resolved as Fig. 3(d), as they have the same coherence lengths $(10.6 \mu \mathrm{m})$. This is, of course, not the case, as non-Gaussian sources generate the classic side lobe artefact. The greater the peak separation in the spectrum is, the greater the number of side lobes and their secondary, and tertiary daughter satellites are. Increasing the number of SLDs in the source will increase the number of side lobes in the A-scan. 


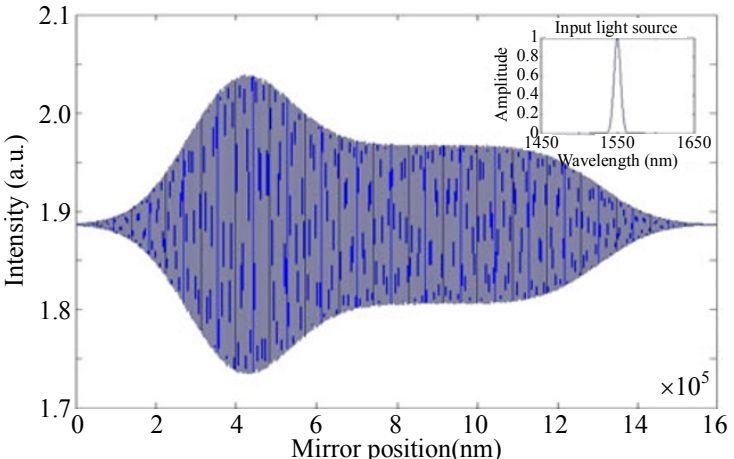

(a)

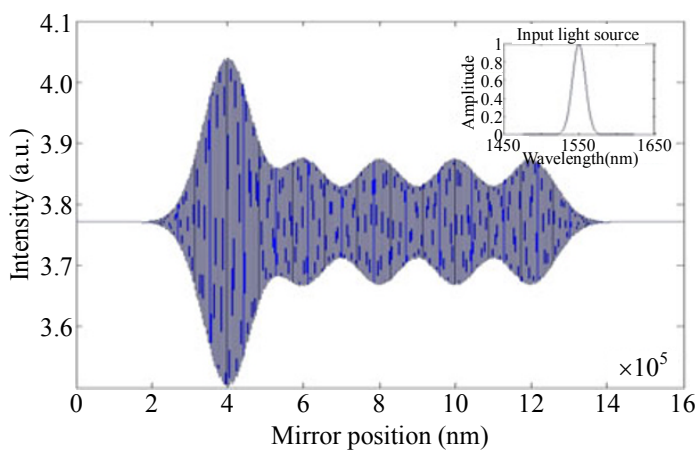

(b)

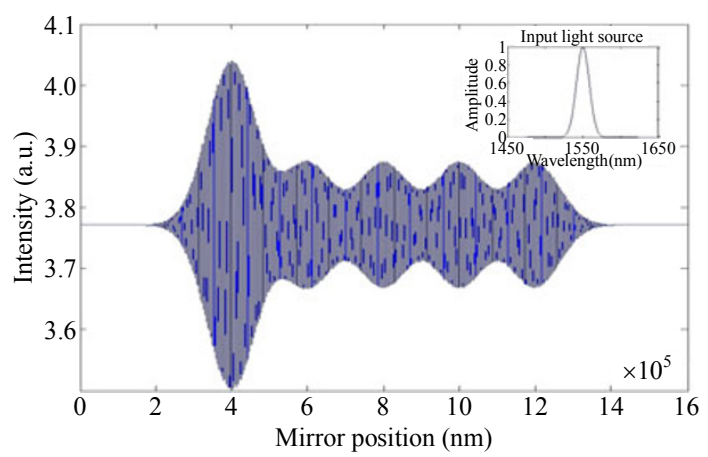

(b)

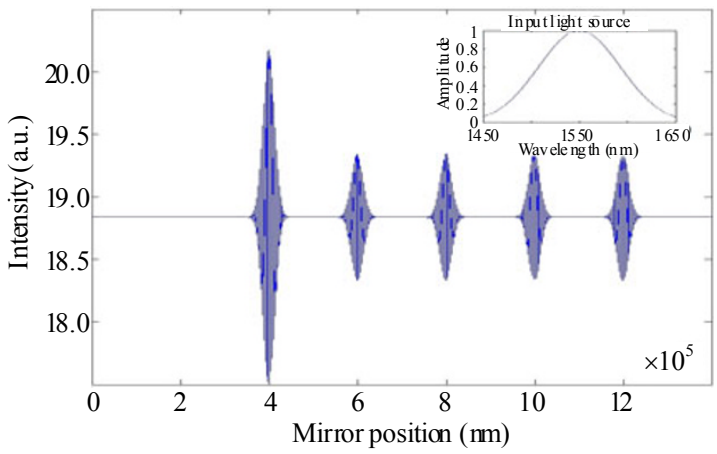

(d)

Fig. 3 OCT interferogram A-scan of a five-layer virtual sample (each layer is $100 \mu \mathrm{m}$ thick) with the associated source spectrum insert: source bandwidths are (a) $10 \mathrm{~nm}$, (b) $20 \mathrm{~nm}$, (c) $50 \mathrm{~nm}$, and (d) $100 \mathrm{~nm}$, with the central source wavelength of $1550 \mathrm{~nm}$.

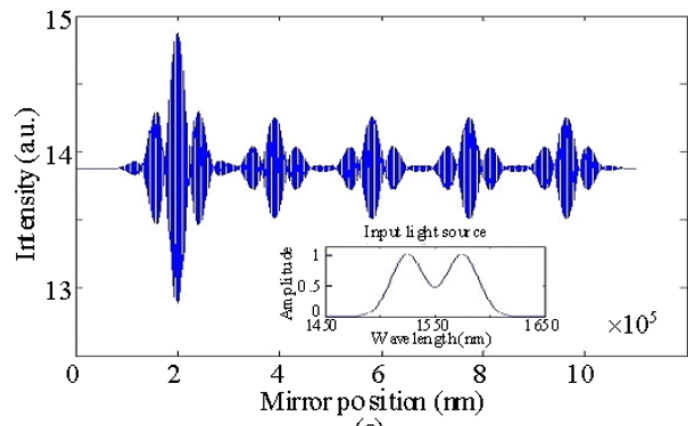

(a)

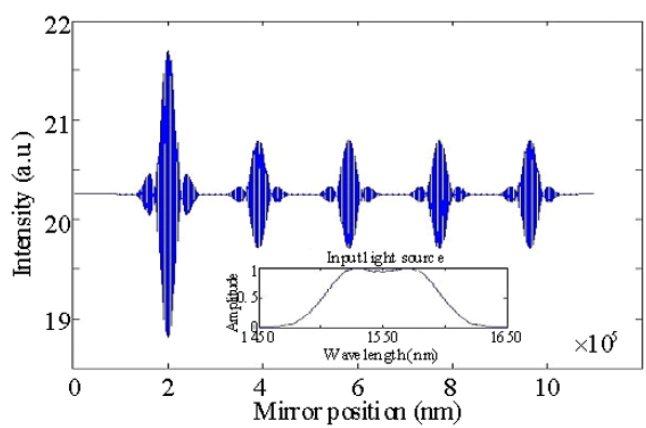

(c)

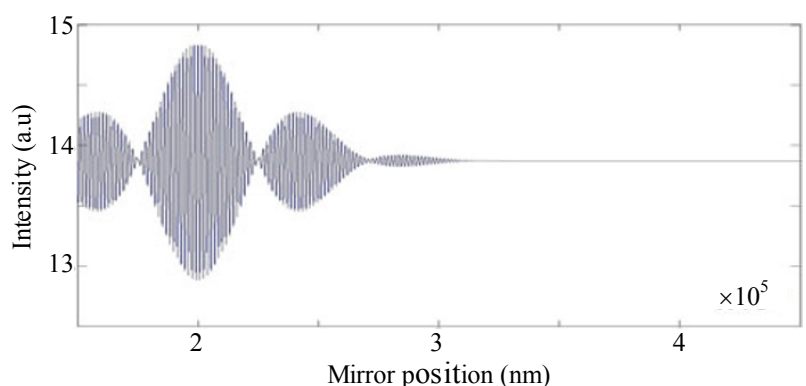

(b)

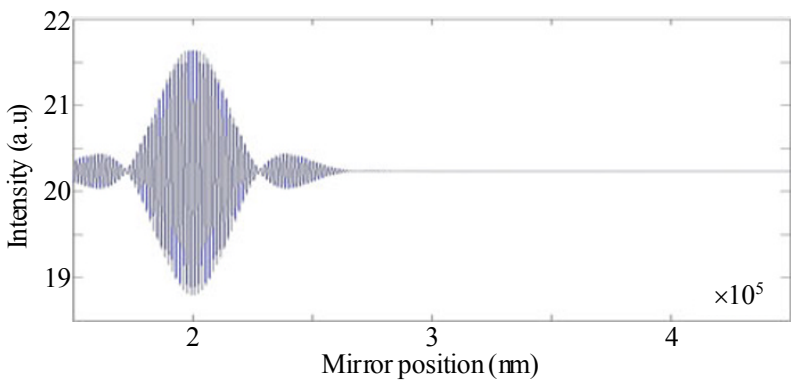

(d)

Fig. 4 OCT interferograms of a five-layer sample [(a) and (c)] and a one-layer sample [(b) and (d)] for a dual SLD source: each individual layer is $95 \mu \mathrm{m}$ thick, the associated dual SLD spectrum is shown as an insert in (a) and (c), and the bandwidth ( $\Delta \lambda) /$ peak amplitude $\left(A_{\max }\right) /$ central wavelength $\left(\lambda_{0}\right)$ combinations for the individual SLD sources are $35 \mathrm{~nm} / 0.9965$ a.u. $/ 1525 \mathrm{~nm}$ and 35 $\mathrm{nm} / 0.9965$ a.u. $/ 1575 \mathrm{~nm}$ for (a) and (b) and $50 \mathrm{~nm} / 0.9266$ a.u. $/ 1525 \mathrm{~nm}$ and $50 \mathrm{~nm} / 0.9266$ a.u. $/ 1575 \mathrm{~nm}$ for (c) and (d). 


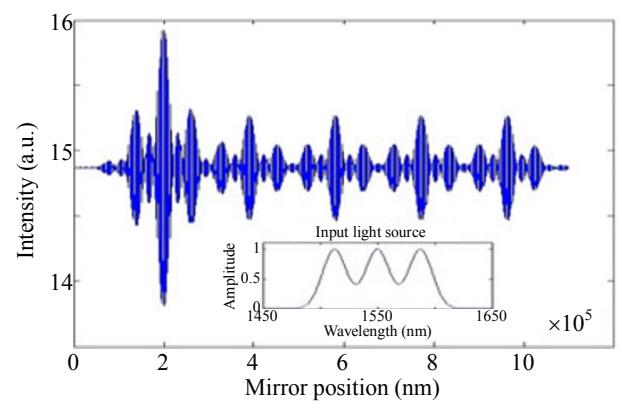

(a)

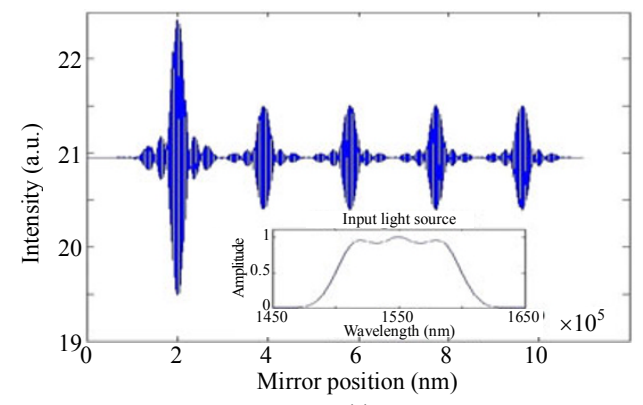

(c)

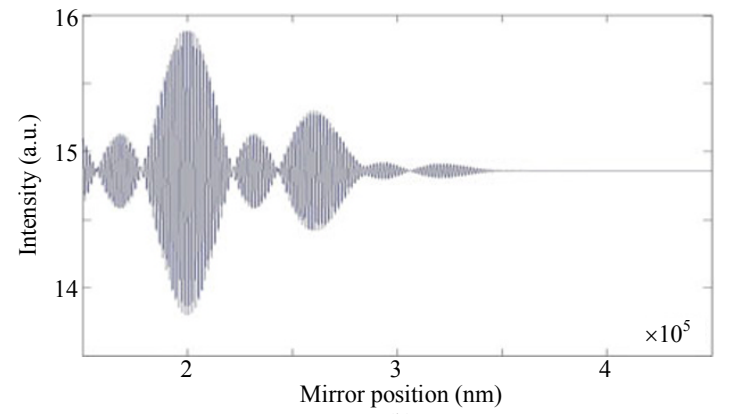

(b)

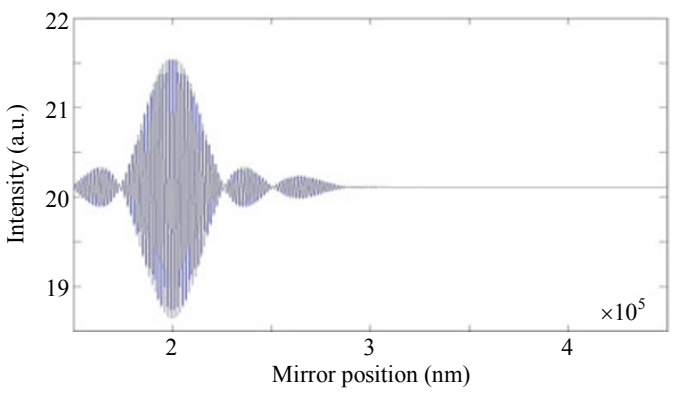

(d)

Fig. 5 OCT interferograms of a five-layer sample [(a) and (c)] and a one-layer sample [(b) and (d)] for a triple SLD source: each individual layer is $95 \mu \mathrm{m}$ thick, the associated triple SLD source spectrum is shown as an insert in (a) \& (c) - for (a) and (b), the individual SLD source's central wavelengths are $1512.5 \mathrm{~nm}, 1550.0 \mathrm{~nm}$ and $1587.5 \mathrm{~nm}$, each with a bandwidth of $25 \mathrm{~nm}$ and an amplitude of $0.9965 \mathrm{a} . u$; for (c) \& (d), the individual SLD source's central wavelengths are $1516 \mathrm{~nm}, 1550 \mathrm{~nm}$ and $1594 \mathrm{~nm}$, each with a bandwidth of $35 \mathrm{~nm}$ and an amplitude of 0.8756 a.u.

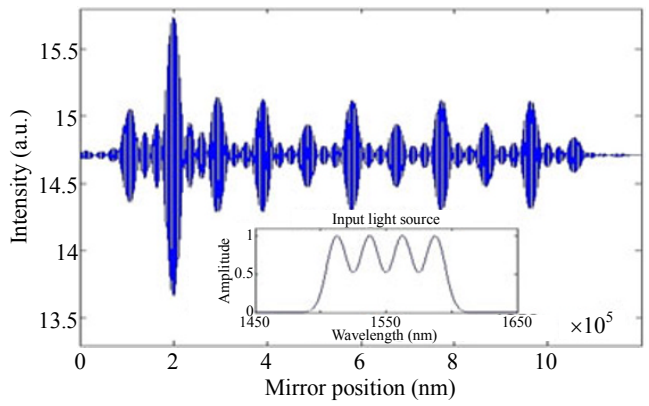

(a)

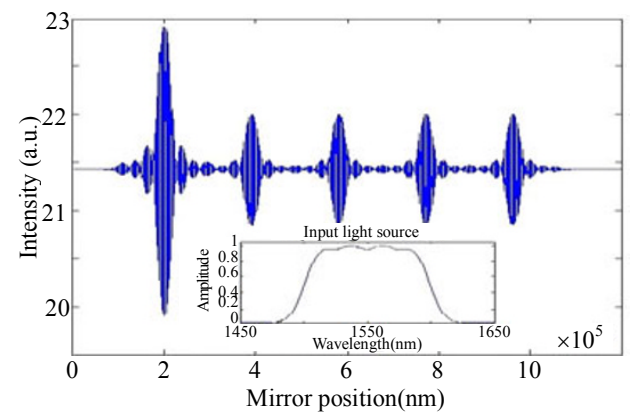

(c)

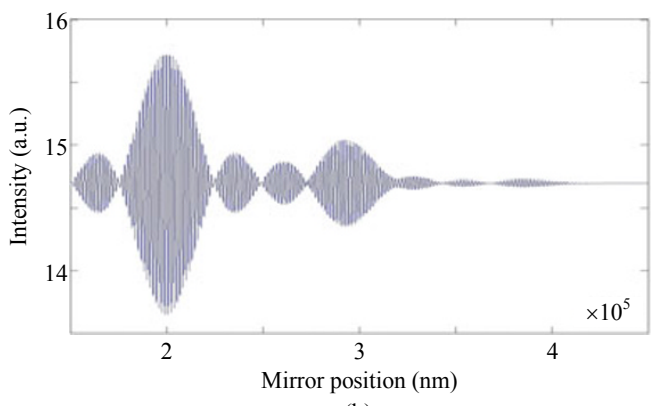

(b)

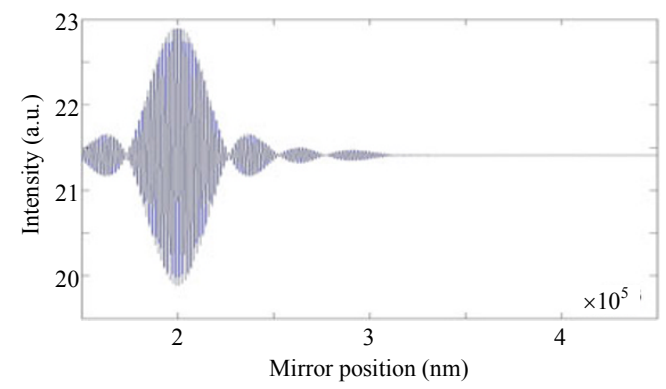

(d)

Fig. 6 OCT interferograms of a five-layer sample [(a) and (c)] and a one-layer sample [(b) and (d)] for a quadruple SLD source: each individual layer is $95 \mu \mathrm{m}$ thick, the associated quadruple SLD source spectrum is shown as an insert in (a) and (c) - for (a) and (b), the individual SLD source's central wavelengths are $1512.5 \mathrm{~nm}, 1537.5 \mathrm{~nm}, 1562.5 \mathrm{~nm}$, and $1587.5 \mathrm{~nm}$, each with a bandwidth of 18 $\mathrm{nm}$ and an amplitude of 0.9908 a.u.; for (c) \& (d), the individual SLD source's central wavelengths are $1512.5 \mathrm{~nm}, 1537.5 \mathrm{~nm}, 1562.5$ $\mathrm{nm}$, and $1587.5 \mathrm{~nm}$, each with a bandwidth of $28 \mathrm{~nm}$ and an amplitude of 0.82 a.u. 
The graphs (a) and (c) in Figs. 4, 5, and 6 demonstrate the issue of non-Gaussian sources. Namely, the side lobes of a strongly reflective layer will appear equal to or greater than the real layer peaks of other layers in the A-scan. How can the detecting software distinguish the real peaks from the artefacts?

The graphs (b) and (d) in Figs. 4, 5, and 6 demonstrate one-layer-sample interferograms, which are effectively inverse Fourier transforms of their associated multi-SLD spectra - the latter inserted in each of the (a) and (c) graphs of Fig. 4, 5 and 6. These (b) and (d) graphs show the extent of side lobe artefacts not present in the 5-layer interferograms of (a) and (c). The (b) and (d) graphs also imply that the 5-layer A-scans are not just showing the contributions of each layer alone. Rather they represent a contribution from the interference signal from the adjacent layer as well. For example, Fig. 6 (b) has 6 detectable side lobes that extend down to the next layer, as will all the other SLD combinations if the layers are close enough. This is in marked contrast to the single SLD source with the similar bandwidth and central wavelength [Fig. 3(d)] as mentioned above. Here, the layers can be as close as a coherence length (10.6 $\mathrm{m})$ and still be distinguishable in the A-scan.

Figures 4-6 show that the number of side lobes increases with the number of tandem SLDs and decreases with the center of each SLD spectrum converge. There is one to two generations of side lobes for the dual-SLD sources (Fig. 4), two to four for the triple-SLD sources (Fig. 5) and three to six for the quad-SLD sources (Fig. 6). Also, the larger the reflection is, the larger the side lobe and satellite magnitudes are.

These complex interferogram results indicate that the side lobes would interact with each other to a greater extent as the layers narrow below $9 L_{\mathrm{C}}$. As has already been mentioned, the result of this is that the phantom layers would appear as strong as the real layers. For example, in Fig. 4(a), if the corresponding secondary peaks of two strata were to be in phase, and collocated, then the sum of this would be equal in magnitude to the primary peak. This phantom layer would appear to be located exactly between the two strata. The complex interaction is also seen in Fig. 6(a), where the secondary peak of the front surface is identical to the main peak of the other layers. The result for the multiple layers shows that this effect is reduced as the separation between the central wavelengths of the sources converge.

\section{Future work}

Future research with this simulation model will explore: (1) modulating the strata thicknesses and their effect on the interferograms and (2) the effect of real OCT light source spectra on their OCT interferogram characteristics. Already the Matlab model's simulation software has presented fresh insights from its capability to generating interferogram "movies". In particular, it can capture a sequence of interferograms as the layer thickness stepwise increases or decreases, showing the change in the layer peak and, where relevant, side lobe peak magnitude and morphology.

The current version of the OCT model is one dimensional. It considers ballistic sample reflections dependent on the refractive index and interface separation. The potential to study the effect of real OCT light source spectra on interferograms is now possible with the next version of the OCT model. The potential to improve the model to study the effect of light sources and ODLs, using realistic virtual samples with additional tissue characteristics, such as absorption, scattering, dispersion, and polarization, is envisaged. This will provide a more realistic understanding of the OCT light source functionality for specific tissue types and conditions.

Due to the ongoing extrinsic evolution of the OCT simulation software, more applications are envisaged. It will be able to forecast misinformation, for which corrections may be able to be made. It 
may also be able to provide insight into choosing the best real OCT light source for a given tissue application. Studying the effect of modulating layer thickness on the interferogram may help in the understanding of new OCT modalities. Its use in physics, biomedical optics, imaging, and photonics education may also be possible.

\section{Conclusions}

We have shown a comparison of broadband single-Gaussian and multi-Gaussian light sources used for OCT. For single-Gaussian sources, the axial resolution improves with the source bandwidth, as expected. However, for narrow bandwidth light sources, their interferograms show overlapping strata peaks. This results in the loss of individual strata information. For an increasing number of multiple-Gaussian sources with the same bandwidth, spectral side lobes increases, reducing the reliability of the A-scan to show accurate layer information without eliminating the side lobes. The more Gaussian the light spectrum is, the more ideal the autocorrelation function is. The potential to use the model to study real OCT light sources such as the SLDs reviewed in this paper, as well as optical delay lines and sample structures, can improve our understanding of the effects that these OCT elements have on the resulting A-scan. With ongoing model development, many more applications are envisaged.

Open Access This article is distributed under the terms of the Creative Commons Attribution License which permits any use, distribution, and reproduction in any medium, provided the original author(s) and source are credited.

\section{References}

[1] A. F. Fercher, "Optical coherence tomography," Journal of Biomedical Optics, vol. 1, no. 2, pp. 157-173, 1996.
[2] J. A. Izatt, M. D. Kulkarni, K. Kobayashi, M. V. Sivak, J. K. Barton, and A. J. Welch, "Optical coherence tomography for biodiagnostics," Optics and Photonics News, vol. 8, no. 5, pp. 41-47, 1997.

[3] W. Drexler, Y. Chen, A. Aguirre, B. Povazay, A. Unterhuber, and J. G. Fujimoto, "Ultrahigh resolution optical coherence tomography," in Optical Coherence Tomography: Technology and Applications, W. Drexler and J.G. Fugimoto, Eds. Berlin: Springer-Verlag, 2008, pp. 241.

[4] P. V. Jansz, G. Wild, S. Richardson, and S. Hinckley, "Simulation of optical delay lines for optical coherence tomography," in Proc. IQEC-CLEO Pacific Rim, Sydney, Aug. 28, pp. 1400-1402, 2011.

[5] P. V. Jansz, G. Wild, and S. Hinckley, "A micro-photonic stationary optical delay line for fiber optic TD OCT," in Proc. OECC ACOFT, Jul. 7-10, pp. 1-2, 2008.

[6] M. Friebel, J. Helfmann, U. Netz, and M. Meinke, "Influence of oxygen saturation on the optical scattering properties of human red blood cells in the spectral range 250 to 2000 nm," Journal of Biomedical Optics, vol. 14, no. 3, pp. 034001-1-034001-6, 2009.

[7] V. R. Shidlovski, Superluminescent diode light sources for OCT, In Optical Coherence Tomogrpahy: Technology and Applications, W. Drexler and J. G. Fugimoto, Eds. Berlin,: Springer-Verlag, 2008, pp. 281-299.

[8] S. G. Adie, "Enhancement of contrast in optical coherence tomography: new modes, methods and technologies," Ph.D. dissertation, School of Electrical Engineering, The University of Western Australia, Perth, Australia, 2007.

[9] A. F. Fercher, W. Drexler, C. K. Hitzenberger, and T. Lasser, "Optical coherence tomography-principles and applications," Report on Progress in Physics, vol. 66, no. 2, pp. 239-303, 2003.

[10] A. V. Zvyagin, M. G. Garcia-Webb, and D. D. Sampson, "Semiconductor line source for low-coherence interferometry," Applied Optics, vol. 40, no. 6, pp. 913-915, 2001.

[11] J. R. Parker, "Optical coherence tomography: endoscopic instrumentation and applications," 2003. Retrieved November 23, 2005, from the Imperial College website: http://www.imperial.ac.uk/ research/photonics/about/staff/james_parker_octrepo rt.pdf.

[12] S. C. Moore, "A theoretical framework for new approaches to scanning and acquisition in optical coherence tomography," Ph.D. dissertation, School of Electrical Engineering, The University of Western Australia, Perth, Australia, 2003.

[13] X. Clivaz, F. Marquis-Weible, R. P. Salathé, R. P. Novàk, and H. H. Gilgen, "High-resolution reflectometry in biological tissues," Optics Letters, vol. 17, no. 1, pp. 4-6, 1992. 
[14] J. M. Schmitt, S. L. Lee, and K. M. Yung, "An optical coherence microscope with enhanced resolving power in thick tissue," Optics Communications, vol. 142, no. 4-6, pp. 203-207, 1997.

[15] W. K. Burns, C. Chen, and R. P. Moeller, "Fiber-optic gyroscopes with broadband sources," Journal of Lightwave Technology, vol. 1, no. 1, pp. 98-105, 1983.

[16] M. Rossetti, A. Markus, A. Fiore, L. Occhi, and C. Velez, "Quantum dot superluminescent diodes emitting at $1.3 \mathrm{~m}$, , IEEE Photonics Technology Letters, vol. 17, no. 3, pp. 540-542, 2005.

[17] D. D. Sampson and W. T. Holloway, "100 mW spectrally-uniform broad band ASE for spectrum-sliced WDM systems," Electronics Letters, vol. 30, no. 19, pp. 1611-1612, 1994.

[18] J. Singh, Semiconductor Devices: An Introduction. New York, USA: McGraw-Hill Inc, 1994, pp. 90.

[19] E. V. Andreeva, P. I. Lapin, V. V. Prokhorov, V. R. Shidlovski, M. V. Shramenko, and S. D. Yakubovich, "Novel superluminescent diodes and SLD-based light sources for optical coherence tomography," in Proc. SPIE, vol. 6627, pp. 662703-1-662703-10, 2007.

[20] J. Wang, M. J. Hamp, and D. T. Cassidy, "Design considerations for asymmetric multiple quantum well broad spectral width superluminescent diodes," IEEE Journal of Quantum Electronics, vol. 44, no. 12, pp. 1256-1262, 2008.

[21] L. H. Li, M. Rossetti, A. Fiore, L. Occhi, and C. Velez, "Wide emission spectrum from superluminescent diodes with chirped quantum dot multilayers," Electronics Letters, vol. 41, no. 1, pp. 41-43, 2005.

[22] P. Bardella, M. Rossetti, and I. Montrosset, "Modelling of broadband chirped quantum-dot super-luminescent diodes," IEEE Journal of Selected Topics in Quantum Electronics, vol. 15, no. 3, pp. 785-791, 2009.

[23] P. D. L. Judson, K. M. Groom, D. T. D. Childs, M. Hopkinson, N. Krstajic, and R. A. Hogg, "Maximising performance of optical coherence tomography systems using a multi-section chirped quantum dot superluminescent diode," Microelectronics Journal, vol. 40, no. 3, pp.
588-591, 2009.

[24] S. K. Ray, K. M. Groom, H. Y. Liu, M. Hopkinson, and R. A. Hogg, "Broad-band superluminescent light emitting diodes incorporating quantum dots in compositionally modulated quantum wells," Japanese Journal of Applied Physics, vol. 45. no. 4A, pp. 2542-2545, 2006.

[25] S. K. Ray, T. L. Choi, K. M. Groom, B. J. Stevens, H. Liu, M. Hopkinson, et al., "High-power and broadband quantum dot superluminescent diodes centered at $1250 \mathrm{~nm}$ for optical coherence tomography," IEEE Journal of Selected Topics in Quantum Electronics, vol. 13, no. 5, pp. 1267-1272, 2007.

[26] A. Somers, W. Kaiser, J. P. Reithmaier, and A. Forchel, "Optical gain properties of InAs/InAlGaAs/InP quantum dash structures with a spectral gain bandwidth of more than $300 \mathrm{~nm}$," Applied Physics Letters, vol. 89, pp. 061107-1-061107-3, 2006.

[27] B. S. Ooi, H. S. Djie, Y. Wang, C. Tan, J. C. M. Hwang, $\mathrm{X}$. Fang, et al., "Quantum dashes on InP substrate for broadband emitter applications," IEEE Journal of Selected Topics in Quantum Electronics, vol. 14, no. 4, pp. 1230-1238, 2008.

[28] A. Baumgartner, C. K. Hitzenberger, E. Ergun, M. Stur, H. Sattmann, W. Drexler, et al., "Resolution-improved dual-beam and standard optical coherence tomography: a comparison," Graefe's Archive Clinical Experimental Ophthalmology, vol. 238, no. 5, pp. 385-392, 2000.

[29] H. Wang, M. W. Jenkins, and A. M. Rollins, "A combined multiple-SLED broadband light source at $1300 \mathrm{~nm}$ for high resolution optical coherence tomography," Optics Communications, vol. 281, no. 7, pp. 1896-1900, 2008.

[30] S. Lee, H. Jeong, and B. Kim, "High-speed spectral domain polarization-sensitive optical coherence tomography using a single camera and an optical switch at $1.3 \mu \mathrm{m}$," Journal of Biomedical Optics, vol. 15, no. 1, pp. 010501-1-010501-3, 2010.

[31] M. J. C. Van Gemert, S. L. Jacques, H. J. C. M. Sterenborg, and W. M. Star, "Skin optics," IEEE Transaction on Biomedical Engineering, vol. 36, no. 12, pp. 1146-1154, 1989. 